\title{
Population-specific diversity of the immunoglobulin constant heavy $G$ chain (IGHG) genes
}

\author{
Arman A. Bashirova ${ }^{1}$, Wanjing Zheng ${ }^{2}$, Marjan Akdag ${ }^{1}$, Danillo G. Augusto ${ }^{3,4}$, Nicolas Vince ${ }^{5}{ }^{5}$, Krista L. Dong ${ }^{6,7}$, Colm O'hUigin $^{1}$ and \\ Mary Carrington (iD) $1,7 \times$
}

(c) The Author(s) 2021

Human immunoglobulin $\mathrm{G}(\mathrm{lg} G)$ molecules, $\lg G 1, \lg G 2$ and $\lg G 3$, exhibit substantial inter-individual variation in their constant heavy chain regions, as discovered by serological methods. This polymorphism is encoded by the IGHG1, IGHG2, and IGHG3 genes and may influence antibody function. We sequenced the coding fragments of these genes in 95 European Americans, 94 African Americans, and 94 Black South Africans. Striking differences were observed between the population groups, including extremely low amino acid sequence variation in IGHG1 among South Africans, and higher IGHG2 and IGHG3 diversity in individuals of African descent compared to individuals of European descent. Molecular definition of the loci illustrates a greater level of allelic polymorphism than previously described, including the presence of common IGHG2 and IGHG3 variants that were indistinguishable serologically. Comparison of our data with the 1000 Genome Project sequences indicates overall agreement between the datasets, although some inaccuracies in the 1000 Genomes Project are likely. These data represent the most comprehensive analysis of IGHG polymorphisms across major populations, which can now be applied to deciphering their functional impact.

Genes \& Immunity (2021) 22:327-334; https://doi.org/10.1038/s41435-021-00156-2

\section{INTRODUCTION}

Human IgG subclasses, IgG1, IgG2, lgG3, and lgG4, display distinct functional properties due to the differences in their constant heavy chains, which consist of $\mathrm{CH} 1, \mathrm{CH} 2$, and $\mathrm{CH} 3$ domains and a hinge region between the $\mathrm{CH} 1$ and $\mathrm{CH} 2$ domains [1]. These fragments contain binding sites for complement component $\mathrm{C} 1 \mathrm{q}$ and Fc gamma receptors $[2,3]$, which regulate antibody effector functions, and the neonatal Fc receptor (FcRn), controlling antibody transport and half-life [4]. The constant heavy chains of the four IgG subclasses are encoded by distinct genetic loci, IGHG1, IGHG2, IGHG3, and IGHG4 (Fig. 1) [5]. These genes form a cluster spanning a $\sim 150 \mathrm{~kb}$ region within the lg heavy chain constant $(I G H C)$ locus on human chromosome 14. The $\mathrm{CH} 1$, hinge, $\mathrm{CH} 2$ and $\mathrm{CH} 3$ domains of each gene are encoded by separate exons. However, IGHG3 is unique in having varying number of hinge exons, ranging from two to four across individuals [6].

Sequence differences in constant heavy chains exist not only between the lgG subclasses, but also within the subclasses, originally discovered using serological methods [7]. Based on antigenic properties, IgG allotypes were classified using the $\mathrm{Gm}$ (Gamma marker) nomenclature, where $\mathrm{G} 1 \mathrm{~m}, \mathrm{G} 2 \mathrm{~m}$, and $\mathrm{G} 3 \mathrm{~m}$ allotypes designate $\lg G 1, \lg G 2$, and $\lg G 3$, respectively. The $\mathrm{Gm}$ allotypes across these three subclasses were observed to be inherited in certain frequent combinations, reflecting a high level of linkage disequilibrium (LD) between the corresponding loci.
Genetic polymorphism underlying the serological Gm diversity was further characterized within each of the IGHG1, IGHG2, and IGHG3 genes [1,7]. Multiple alleles of these genes are annotated in the IMGT database, including 14 IGHG1, 17 IGHG2, and 29 IGHG3 alleles encoding 6,7 , and 21 distinct protein variants, respectively, as of August 2021 (www.imgt.org). Differences between alleles include single nucleotide polymorphisms (SNPs) as well as variation in the number of IGHG3 hinge exons. These data show that IGHG variation involves more amino acid positions than those identified serologically.

Functional consequences of IGHG polymorphism are largely unknown, but a few have been elucidated. An IGHG3 variant with histidine at position $435(435 \mathrm{H})$ in the $\mathrm{CH} 3$ domain was shown to have prolonged half-life compared to that containing the arginine variant $(435 \mathrm{R})$ due to higher affinity of the lgG3-435H variant to FcRn at low pH [4]. Differential FcRn binding has also been detected for IgG1 allotypes, which are fixed for the $435 \mathrm{H}$ variant, but differ from one another at positions $214(\mathrm{CH} 1)$ and/or 356/358 (CH3) [8]. Polymorphisms at residues 291, 292 and 296 in the lgG3 $\mathrm{CH} 2$ domains have recently been shown to influence antibody dependent cellular cytotoxicity (ADCC) in a comprehensive study that examined 27 genetically defined IgG allotypes [9]. In addition, antibody effector functions can be modulated by length of the IgG3 hinge $[9,10]$. Besides the direct effect of allotypic variation on the antibody structure and function, certain variants in the $\mathrm{CH}$

\footnotetext{
${ }^{1}$ Basic Science Program, Frederick National Laboratory for Cancer Research in the Laboratory of Integrative Cancer Immunology, National Cancer Institute, Bethesda, MD, USA ${ }^{2}$ The Laboratory of Integrative Cancer Immunology, National Cancer Institute, Bethesda, MD, USA. ${ }^{3}$ Programa de Pós-Graduação em Genética, Universidade Federal do Paraná, Curitiba, Brazil. ${ }^{4}$ Department of Neurology, University of California San Francisco, San Francisco, CA, USA. ${ }^{5}$ Université de Nantes, CHU Nantes, Inserm, Centre de Recherche en Transplantation et Immunologie, UMR 1064, ITUN, F-44000 Nantes, France. ${ }^{6}$ Females Rising through Education, Support, and Health, Durban, KwaZulu-Natal, South Africa. ${ }^{7}$ Ragon Institute of MGH, MIT and Harvard, Cambridge, MA, USA. ${ }^{凶}$ email: carringm@mail.nih.gov
} 


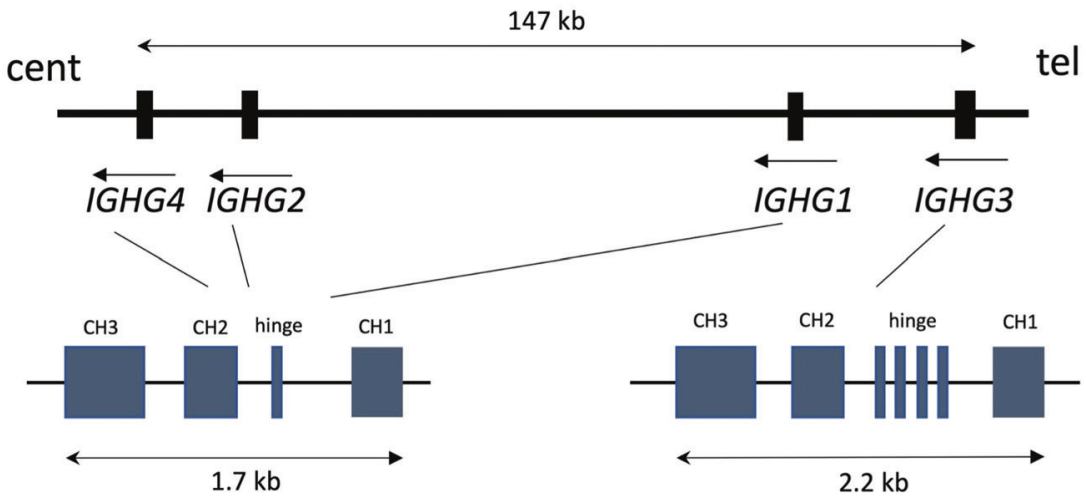

Fig. 1 Schematic map of the IGHG locus. The IGHG genes have similar exon/intron structure, except for IGHG3, which has multiple hinge exons. The map is drawn based on the human genome assembly (GRCh38.p13) and transcript data extracted using the Ensembl browser (www.ensembl.org).

domains may be in LD with polymorphisms that regulate IgG subclass switching, possibly explaining the variation in IgG subclass serum levels observed in carriers with certain lgG allotypes [1, 11-13].

The biological importance of IGHG polymorphism is suggested by multiple disease associations with this locus [14-16]. From a clinical standpoint, the potential immunogenicity of therapeutic antibodies when their allotypes differ from the patient's endogenous allotypes may result in poor response to these therapies, although evidence is lacking in this regard [1].

lgG allotypes have been studied extensively using serological methods and were found to display population-specific frequency distributions [7, 17, 18]. However, the corresponding genetic information is limited. A recent study by Calonga-Solis et al. [19] described polymorphism in the IGHG genes in Brazilian populations, including Amerindians, Japanese- and Euro-descendants, but notably, people of African descent were not considered. Here, we characterized IGHG1, IGHG2, and IGHG3 coding regions polymorphism in three population groups, including African Americans (AA, $N=94$ ), European Americans (EA, $N=95$ ) and Black South Africans ( $S A, N=94)$. As a validation, we compared our genotyping data with that available from the 1000 Genomes Project (1kGP). These data lay a foundation for characterizing the functional consequences for IGHG variation and its potential impact on disease and therapy outcomes.

\section{RESULTS}

\section{IGHG SNPs and haplotypes}

We amplified IGHG1, IGHG2, and IGHG3 genomic fragments, including exons encoding the $\mathrm{CH} 1$, hinge, $\mathrm{CH} 2$ and $\mathrm{CH} 3$ domains (Fig. 1), and sequenced the PCR products using Sanger methodology in order to identify polymorphic sites. The fragment encompassing the IGHG3 hinge exons was amplified using primers outside of the $\sim 200 \mathrm{bp}$ repetitive element (Fig. S1) and the number of exons in each person was determined based on electrophoretically-defined PCR fragment length. SNPs in the IGHG3 hinge exons were not considered because of the inability to read sequences directly upon heterozygosity for exon copy number. Our analysis also did not include the IGHG4 gene given the previously reported copy number variation at this locus [20].

Sequence analysis of the coding regions in the three genes revealed a total of 87 SNPs across the three populations sampled in our study (Table S1). These data were compared to publicly available whole genome sequences by extracting variants in corresponding regions from two $1 \mathrm{kGP}$ datasets: phase 3 [21] (1 kGP-ph3) and the newly assembled high coverage version, $1 \mathrm{kGP}-30 \mathrm{X}$ [22]. Of 87 SNPs detected in our cohorts, 71 were present in $1 \mathrm{kGP}-\mathrm{ph} 3$, and 83 were detected in $1 \mathrm{kGP}-30 \mathrm{X}$
(Table S1). While the vast majority of the SNPs that were present in one dataset and not the other were of low frequency, there were several common SNPs that were discrepant in this regard (Table S1). For example, variants at positions 161 and 311 in $\mathrm{CH} 3$ exons of IGHG2 and IGHG1, respectively, were present in our dataset and $1 \mathrm{kGP}-30 \mathrm{X}$ (16-29\% allelic frequency), but absent in 1kGP-ph3. On the other hand, 1kGP-30X detected SNPs at positions 168 and 313 of IGHG1 CH3 and IGHG2 $\mathrm{CH} 3$, respectively (19-24\% allelic frequency), and these SNPs were not found in our dataset nor $1 \mathrm{kGP}$-ph3. The possibility of errors in the 1kGP datasets with regard to common SNPs is evident when comparing $1 \mathrm{kGP}$ data to that in the IMGT for IGHG alleles, which represent CDNA clones deposited by various research groups (Fig. S2). The common SNPs missed in $1 \mathrm{kGP}$-ph3 and detected in our cohorts are present among the IMGT alleles. Further, SNPs present in $1 \mathrm{kGP}-30 \mathrm{X}$ with frequency $>3 \%$, but missing in our data are also missing in IMGT. Thus, our genotypes more closely reflect those compiled in the IMGT dataset than does either version of the $1 \mathrm{kGP}$ datasets.

We used Haploview software to phase the identified SNPs and predict IGHG alleles, which we then matched to alleles annotated in the IMGT database (Tables S2-S4). Several putatively new alleles were identified, with allelic frequencies of up to $12 \%$. Alleles that were very common in one population were often much rarer in another; for example, IGHG1*03,IGHG2*03, and IGHG3*11 were common in EA, but less so in AA and $\mathrm{SA}$, and IGHG1*02, IGHG2*06, and IGHG3*01 were common in AA and SA, but less so (or missing altogether) in EA. Thus, IGHG allelic frequencies demonstrated remarkable population-specific distributions.

\section{IGHG allotypes}

IGHG allotypes (i.e., protein variants) were estimated by phasing only amino acid changing SNPs in our dataset. In order to compare our data to the $1 \mathrm{kGP}$ data, we extracted phased nonsynonymous IGHG SNP data from 1kGP-ph3 for three superpopulations, African (AFR, $N=661$ ), European (EUR, $N=503$ ), and East Asian (EAS, $N=504$ ), and considered variants present with frequencies $>1 \%$. As indicated above, a number of discrepancies were evident between both versions of the $1 \mathrm{kGP}$ data and our data. We focused here only on the older 1kGP-ph3 because the $1 \mathrm{kGP}-30 \mathrm{X}$ data contains several SNPs with high frequencies that are likely to have been assigned to IGHG genes inaccurately, and it is preferable to analyze a dataset that may miss some SNPs rather than one that contains falsely assigned variants. Alleles encoding the same amino acid sequence (i.e., alleles that differed only by synonymous changes) were grouped together and given an allotype name that corresponds to the most frequent allele in that grouping. For instance, the allotype $\mid \mathrm{GHG} 1^{*} 02$ is encoded by multiple IGHG1 alleles that differ only by synonymous changes $\left(\right.$ IGHG ${ }^{*} 02,{ }^{*} 05,{ }^{*} 09$, etc.; Table S2). 


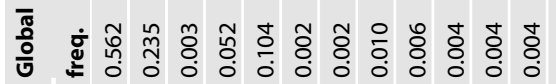

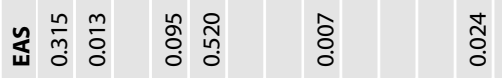

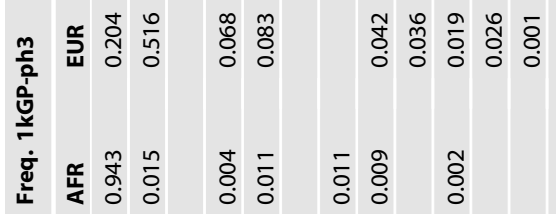

ธุ

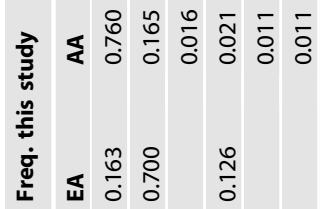

บุ $>$

$\ll<\ll$

$\lessgtr \geq>$

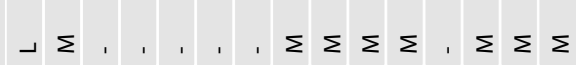

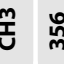

○

ลิ

บิ

$\stackrel{\text { ஸे }}{\text { ก }}$

동

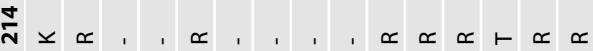

ล

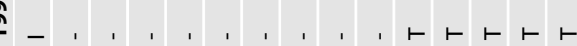

$\bar{m}$

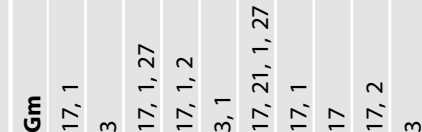

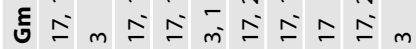

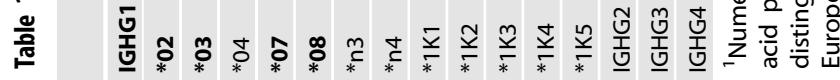

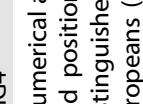

The most frequent IGHG1 allotypes differed across population groups: $\mid \mathrm{GHG} 1^{*} 03$, IGHG1*02, and $\mid \mathrm{GHG} 1 * 08$, in individuals of European descent (EA and EUR; IED), individuals of African descent (AA, SA and AFR; IAD), and EAS, respectively (Table 1). Strikingly, IGHG1*02 was nearly fixed in the SA sample and it was also the most common IGHG1 variant across populations overall. The most frequent EAS allotype, IGHG1*08, could be a product of allelic gene conversion between IGHG1*02 and IGHG1*03 (Table 1).

IGHG2 allotypes demonstrated higher level of diversity than IGHG1 with IGHG2*02, IGHG2*03 and IGHG2*06 observed most frequently (Table 2). While IGHG2*03 was common in all six populations, IGHG2*02 was relatively rare among IAD and IGHG3*06 was absent among IED. A new allotype, IGHG2*n3, was identified in the $\mathrm{AA}$ and $\mathrm{SA}$ populations at fairly high frequencies, but was strikingly absent in the AFR sample of $1 \mathrm{kGP}$; this allotype differs from IGHG2*03 by a single amino acid at position 422 in the $\mathrm{CH} 3$ domain (V/l, Table 2), and is likely to be a product of gene conversion between IGHG2 and IGHG3 (Tables 2 and 3). The absence of IGHG2*n3 in the $1 \mathrm{kGP}$-ph3 could be a result of incorrect sequence assembly, which did not identify the $422 \mathrm{~V} / \mathrm{I}$ polymorphism at this locus (G2_CH3_243, Table S1).

A single trimorphism (V/L/M) was detected at position 309 of IGHG2 (G2_CH2_231), where the 309M variant was found exclusively in SA. Interestingly, one SA individual possessed three nucleotide variants at the corresponding position, suggesting a gene duplication of the IGHG2 locus (Fig. S3). Although the gene duplication may have been present in more individuals, this is likely to be a rare event since the SNP data were, overall, consistent with Hardy-Weinberg equilibrium.

The IGHG3 allotypes demonstrated the highest level of diversity among the three loci (Table 3). IGHG3*11 is the most common allotype in IED, whereas IGHG3*01 is the most common allotype in IAD and EAS. These two allotypes differ only at position 296 (Y/F) and cannot be distinguished serologically. IGHG3*03 was not present in the 1kGP-ph3 dataset, which misses SNP encoding the IGHG3 419Q/E polymorphism, likely due to sequence assembly error (G3_CH3_234, Table S3).

The IGHG3 allotypes containing histidine at position 435 , which is known to increase the lgG3 half-life and facilitate placental transport [4], were rare in our dataset (IGHG3*17 and IGHG3*n5). The corresponding SNP rs4042056 (G3_CH3_283) was not detected in our EA sample (i.e., fixed for arginine at this position), but was present at frequencies of $3 \%$ and $10 \%$ among the $\mathrm{AA}$ and SA individuals, respectively (Table S1). This SNP was detected at higher frequency in the 1kGP-ph3 dataset (10-16\% in EUR, AFR and EAS) giving rise to multiple haplotypes (Table 3 and S5).

The presence of four IGHG3 hinge exons was by far the most common length variant across the genotyped populations followed by presence of three exons, which showed higher allelic frequency in AA/SA compared to EA (Table 4). We have also detected single individuals carrying an allele with two exons and an allele with five exons in an AA and an EA individual, respectively. For the majority of allotypes identified in our study, the hinge length can be unambiguously assigned using the known IMGT alleles and the $\mathrm{CH}$ domain sequences. The exception is distinguishing IGHG3*11 from IGHG3*12, which are identical in the $\mathrm{CH}$ domains, but possess hinge domains encoded by four vs. three exons, respectively.

Overall, frequencies of the allotypes encoded by the three IGHG genes demonstrated a high level of diversity across populations, and support genetic admixture in the AA population. While providing new details, the data were consistent with previous serological and genotypic findings.

\section{LD between the IGHG genes}

Serological data implicate a high level of LD between the IGHG genes [7], and to test this in our dataset, multigenic haplotypes 
นิ氏

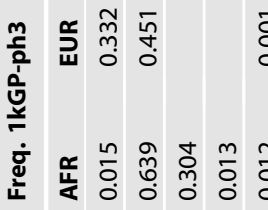

$\frac{\text { 응 }}{0}$

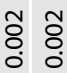

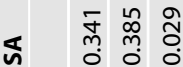

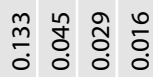

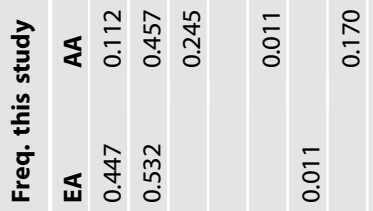

สู

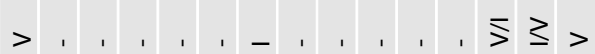

$\frac{8}{2}$

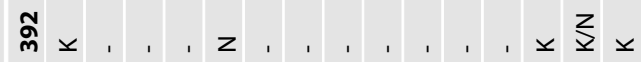

$\stackrel{m}{\underline{x}}$

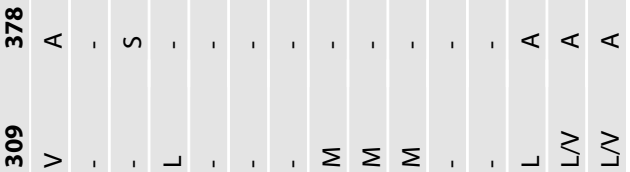

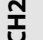

: $\Sigma=>>>,>>>,>>>>$

$\frac{m}{N}$

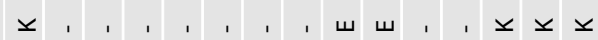

$\stackrel{\varrho}{0}$ $\stackrel{\Perp}{5}$

ปิ $z$ $\sum n$

폰

$\stackrel{\infty}{\circ} \circ 0$ a a a, a a a a a, a a a

กิ

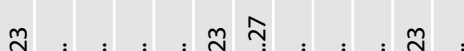

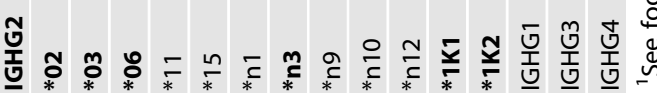

were generated using Haploview based on amino acid changing variants (Fig. 2, S4 and Tables S6-S7). There were 10 haplotypes in the EA group and 18 haplotypes in each of the AA and SA groups restricting to those observed at frequency of $>1 \%$. Haplotype frequencies differed substantially between population samples with no common haplotypes observed between EA and SA except for one (Fig. 2A, Table S6). This is a reflection of the differential allotype frequencies across the groups. Nine haplotypes were shared by the AA and SA groups, and four haplotypes were shared between $A A$ and $E A$, highlighting admixture between these two groups. The haplotypes were further categorized using serological Gm nomenclature [7] (Fig. 2B, Table S7). The corresponding frequencies were concordant with previously published European and South African data $[7,17,18]$.

\section{Inter-population differentiation}

The apparent population-specific distribution of IGHG alleles prompted us to evaluate their differentiation by calculating fixation indices $\left(F_{S T}\right)$, which measures inter-population variance of allele frequencies over an average diversity of populations [23]. Large $F_{S T}$ values indicate inter-population differentiation and suggest the possibility of natural selection. We calculated pairwise $\mathrm{F}_{\mathrm{ST}}$ of IGHG1, IGHG2, and IGHG3 non-synonymous SNPs with global minor allele frequencies $>10 \%$ in the AFR, EUR and EAS superpopulations from $1 \mathrm{kGP}$-ph3 and compared them with the value distribution of a genome-wide sample of SNPs in the same populations (Fig. S5). Four SNPs demonstrated significantly high $\mathrm{F}_{\mathrm{ST}}$ values in pairwise comparisons (Table 5). Whereas all four SNPs showed significantly high $\mathrm{F}_{\mathrm{ST}}$ values in the AFR-EUR comparisons, none were significant in the AFR-EAS analysis; three of the four were significantly high in the EUR-EAS comparison. The $\mathrm{F}_{\mathrm{ST}}$ pattern is consistent with the breakdown of the strong LD between the IGHG1 SNPs encoding amino acid positions 214, 356 and 358 in the EAS super-population. Strong LD across these three amino acids in IGHG1 is characteristic of IED and IAD, whereas breakdown between positions 214 and 356 forming the common IGHG1*08 allotype is characteristic of EAS (Table 1).

\section{DISCUSSION}

IgG allotype diversity has been characterized across various populations by serological methods $[7,17,18]$, whereas characterization of polymorphisms and their frequencies in the corresponding IGHG genes remains sparse due to the homology between the genes and related difficulties in analyzing genotypes. Here, we determined IGHG variation in three population groups from the U.S. and South Africa, representing the first comprehensive genetic analysis of the IGHG locus in IAD, and compared these to the $1 \mathrm{kGP}$ data. Overall, our data were in agreement with the $1 \mathrm{kGP}$ data, but certain distinctions were observed. These included a few common SNP discrepancies between the two datasets and greater diversity of IGHG alleles in the $1 \mathrm{kGP}$ dataset than in ours. There are likely several causes of these discrepancies. The $1 \mathrm{kGP}$ data is obtained using next generation sequencing (NGS), which relies on assembly of short overlapping reads $(\sim 100 \mathrm{bp})$ that can phase variants inaccurately [24], particularly in regions of high sequence similarity, such as the IGHG genes. These issues probably resulted in drop-out of low-confidence SNPs in 1kGP-ph3, and erroneous mapping in the less conservative approach of $1 \mathrm{kGP}$ $30 \mathrm{X}$. Although our data may be prone to allele drop-out if a variant occurs within the site of primer annealing, it is strengthened by sequencing of long genomic fragments (600-1800 bp) derived from gene-specific amplification, which increases confidence in accuracy of gene-specific calling of the variants. Of note, with regard to common SNPs that were discrepant between our data and the $1 \mathrm{kGP}$ datasets, we found that our frequencies were generally closer to that of gnomAD data generated by NGS (http://www.ensembl.org). Further, population substructure 


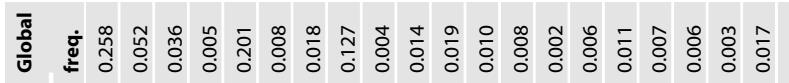

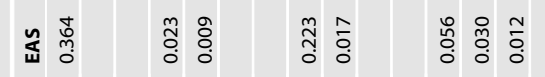

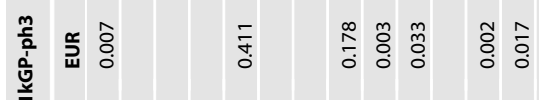

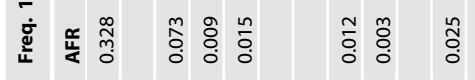

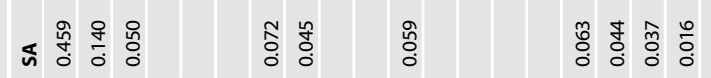

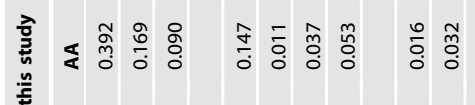

它 :

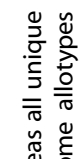

离

$\stackrel{\circ}{\dddot{2}}$

ชฺุ

:̊․:

낭

$\stackrel{\mathscr{\gamma}}{\alpha}$

穿部

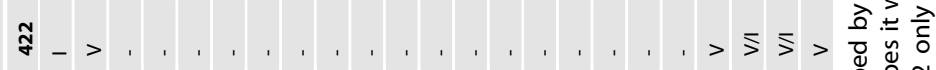

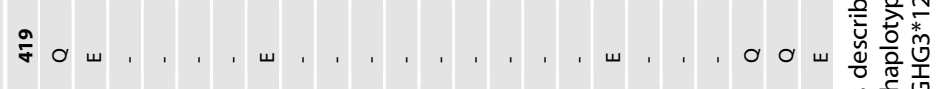

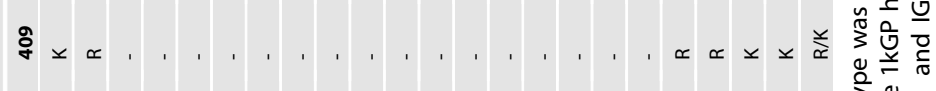

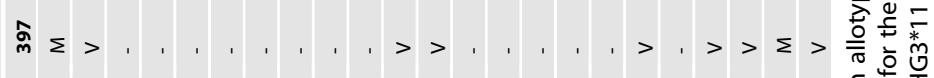

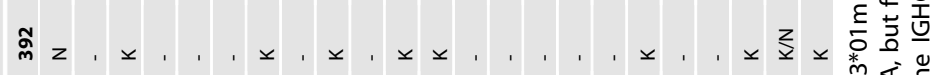

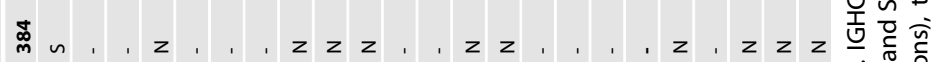

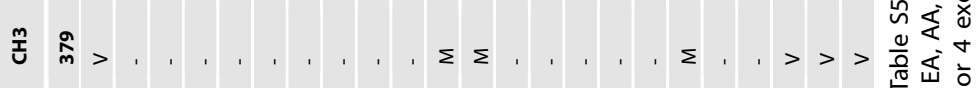

贾

$\widehat{\tilde{m}}<\ldots, \ldots<0$ ०

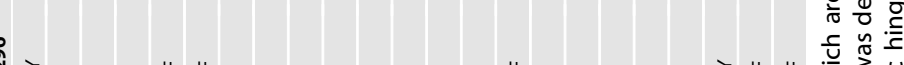

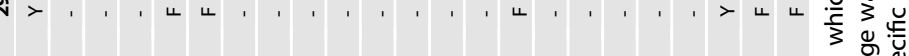

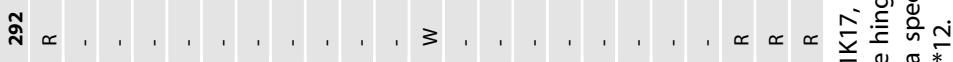

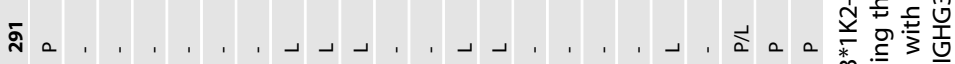

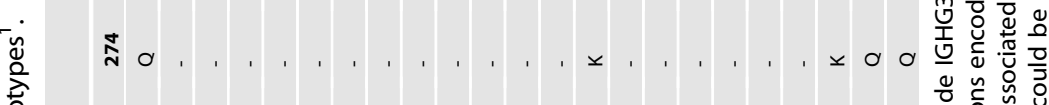

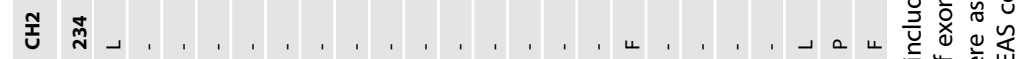

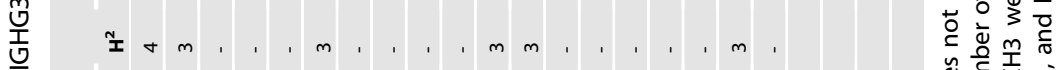

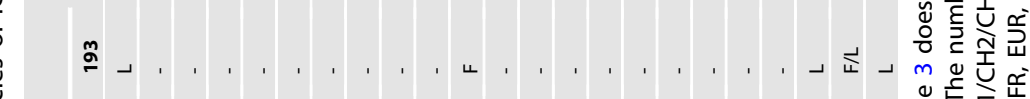

도도.

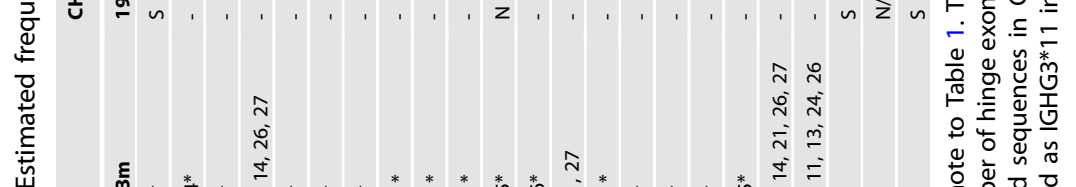

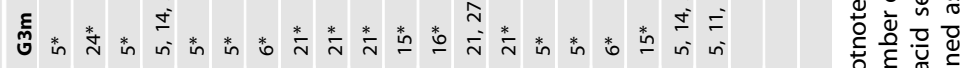

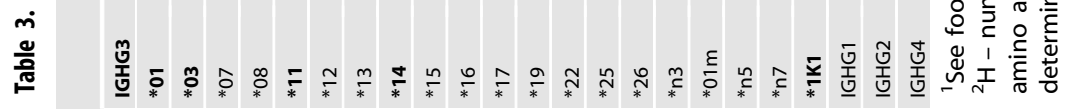


differentiating EUR from EA, or AFR from AA and SA may impact genotypic differences across these populations. Additional limitations of our study that could have affected the data include relatively small sample sizes that would miss rare variants as well as potential population substructure within the groups that may influence haplotype estimation. However, the consistency of our data with Hardy-Weinberg equilibrium, and its general concurrence with $1 \mathrm{kGP}$ data, IMGT allelic variation, and published serological data point to accuracy in our genotyping data of the IGHG genes. Importantly, these consistencies endorse the authenticity of the extended information regarding genetic variation at the IGHG locus provided by our genotyping.

Some differences observed across populations involve variants that may influence antibody function. The IGHG3 $435 \mathrm{H}$ variant, which associates with prolonged half-life of the antibody due to efficient FcRn binding [4], has been found at higher frequency in IAD and EAS compared to IED. The most frequent IGHGI allotype IGHG1*08 in EAS corresponds to $\mathrm{Gm} 3,1$, which showed the strongest interaction with FcRn [8], whereas the most frequent IGHG1 allotypes in IAD and IED correspond to the intermediate

Table 4. IGHG3 hinge exon copy number variation (allele frequency).

\begin{tabular}{|lllll|} 
\# hinge exons & Length, aa & $\begin{array}{l}\mathbf{E A} \\
(\mathbf{N}=\mathbf{9 5})\end{array}$ & $\begin{array}{l}\mathbf{A A} \\
(\boldsymbol{N}=\mathbf{9 4})\end{array}$ & $\begin{array}{l}\text { SA } \\
(\boldsymbol{N}=\mathbf{9 4})\end{array}$ \\
\hline 2 & 32 & & 0.005 & \\
\hline 3 & 47 & 0.037 & 0.234 & 0.239 \\
\hline 4 & 62 & 0.958 & 0.761 & 0.761 \\
\hline 5 & 77 & 0.005 & & \\
\hline
\end{tabular}

and weaker binders, Gm17,1 (IGHG1*02) and Gm3 (IGHG1*03), respectively (Table 1 ). IGHG $1 * 02$ and IGHG $1 * 03$ also differentially bind to decoy FcRs from HSV-1 [25] and HCMV [26], which may potentially underlie disease associations $[15,16]$. IGHG3 allotypes with a leucine at position 291 (291L), a tryptophan at position 292 $(292 \mathrm{~W})$, and a phenylalanine at position 296 (296F) have been found to exhibit reduced ADCC [9]. The 291L and 296F allotypes were present at higher frequencies in IED than in IAD, and the 292W was detected only in 1kGP-ph3 data at low frequencies in EUR and EAS. Given the observed frequency patterns, IAD may possess more potent IgG3 allotypes than IED. Notably, among the functionally distinct allotypes are those defined by SNPs that demonstrated significant inter-population differentiation by $F_{S T}$ analysis (Table 5), suggesting that these SNPs may be under selection pressure due to their functional consequences.

Another potentially functional polymorphism that is differentially present across populations, is the IGHG3 hinge length variation. The most common variant is encoded by four exons, and the second most common variant is encoded by three exons, which is present more frequently among IAD than IED (Table 4). Chu et al. [10] suggested that decreasing the hinge length diminishes phagocytic activity of lgG3 based on studies using hinge variants on the backbone of the IGHG3*01 allotype. However, our genotyping data indicate that the three-exons variant does not occur on a IGHG3*01 backbone, but rather, is present primarily in an IGHG3*03 allotype (Table 3). De Taye et al. [9] tested only natural variants for ADCC activity and did not detect clear difference between three- and four-exon hinge allotypes, but the IGHG3*04 allotype with shorter hinge, encoded by two exons, demonstrated higher ADCC capacity compared to other IGHG3 allotypes. This allotype has been observed only in one individual in our dataset. Further, the
A

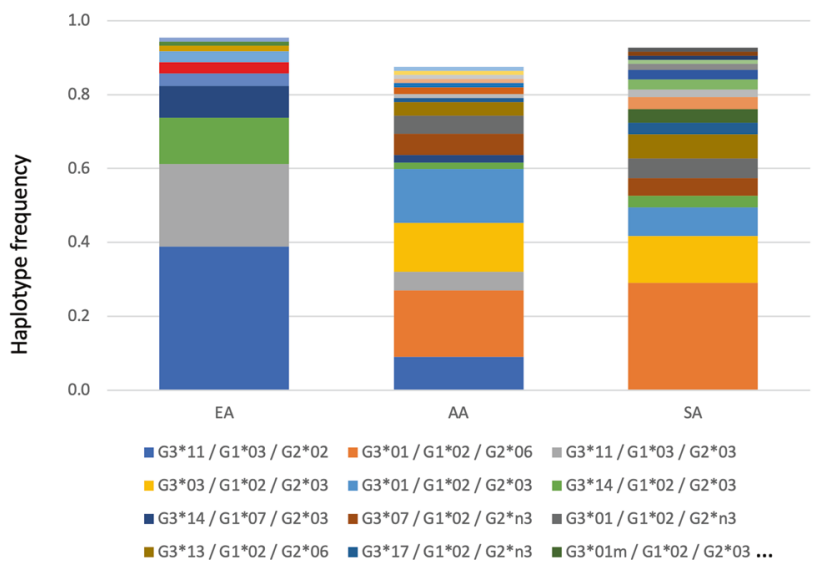

Fig. 2 IGHG3_IGHG1_IGHG2 haplotypes estimated in three populations with frequency $>1 \%$. A Haplotypes estimated using genetic data of non-synonymous SNPs. Allotypes for each locus represent unique amino acid sequences as shown in Tables 1-3. B Gm haplotypes corresponding to the genetic haplotypes according to Lefranc and Lefranc [7]. The data for these graphs is shown in Tables S6 and S7.

Table 5. Non-synonymous IGHG SNPs with significantly high $\mathrm{F}_{\mathrm{ST}}$ values in pairwise analysis of the AFR, EUR and EAS populations from the $1 \mathrm{kGP}$ data?

\begin{tabular}{|c|c|c|c|c|c|c|}
\hline rs \# & IGHG & AA pos. (Eu) & Assoc. alleles & $F_{S T}$ AFR-EUR & $F_{S T}$ AFR-EAS & $F_{S T}$ EUR-EAS \\
\hline rs1071803 & IGHG1 & 214 & ${ }^{*} 03 / 08$ & 0.628 & 0.5514 & 0.01 \\
\hline rs1045853 & IGHG1 & 356 & ${ }^{*} 03$ & 0.623 & 0 & 0.606 \\
\hline rs11621259 & IGHG1 & 358 & ${ }^{*} 03$ & 0.625 & 0 & 0.607 \\
\hline rs12890621 & IGHG3 & 296 & *11/12 & 0.619 & 0.0011 & 0.607 \\
\hline
\end{tabular}

${ }^{1}$ In bold - significant $F_{\mathrm{ST}}$ values. 
IGHG3*17 and IGHG3*01m versions of an anti-HIV broadly neutralizing antibody with hinges encoded by three and four exons respectively, did not show consistent differences in Fcmediated effector functions nor viral neutralization [27]. On the other hand, mutant IGHG3 hinge variants resembling the natural two and three exon allotypes were shown to enhance complement activation and complement-mediated cell lysis as compared to the four exon variant $[28,29]$. Thus, functional distinctions between the hinge length variants are plausible, but more work needs to be done with regard to naturally occurring variants.

Multiple disease associations with IgG allotype polymorphisms have been reported in the literature, supporting differential functions of IgG allotypes [14-16]. Given the high level of interpopulation diversity at the locus, it is critically important to perform IGHG disease association studies in cohorts of sufficiently large sizes and account for population substructure using genome-wide SNP data. The IGHG locus is consistently excluded from SNP-based genome-wide association studies (GWAS) due to difficulties in distinguishing the homologous IGHG genes, which like the HLA class I loci, contain polymorphic sites potentially acquired by gene conversion. Therefore, possible associations with this locus would be missed in these studies. The analysis of the IGHG locus should be feasible, however, with the increasing volume of whole exome/genome sequencing data and cautious attention to accurate assembly of sequences. Comparison of data obtained using different methods, as performed in this study, is important for validation and improvement of the genotyping protocols and analyses. This may require parallel analyses of the IGHG locus in a larger cohort by both Sanger sequencing and whole exome/genome NGS with proper correction for population stratification, missing in the current study. While Sanger data can improve phasing of short NGS reads, NGS data may help to determine whether primer-annealing regions contain SNPs in Sanger sequencing. These data can be utilized further to develop targeted NGS protocols for IGHG typing. In addition, it would be useful to check whether SNP data obtained using genome-wide chips can be applied to impute IGHG alleles similar to that for HLA genes [30]. This would allow interrogation of the locus in existing and future GWAS. Alternative non-PCR based approaches to analyze long genetic fragments, such as fosmid cloning or nanopore sequencing, may provide the most accurate data, but these methods are difficult to apply in large cohorts.

In summary, we have characterized IGHG variation in three distinct populations using gene-specific PCR amplification and Sanger sequencing. Despite its limitations, this work represents a next step towards better understanding of the IGHG diversity. Deeper knowledge of the IGHG variation and its physiological consequences will provide further insight into understanding antibody function and promote therapy design in the context of population diversity.

\section{MATERIAL AND METHODS \\ Human subjects}

Blood samples from healthy African American donors $(N=94)$ were obtained previously from a collaboration with the Duke CHAVI 008 A study (Al067854) [31]. Samples from healthy donors of European ancestry $(N=$ 95) were derived from the Research Donor Program (RDP) at the Frederick National Laboratory for Cancer Research. South African samples $(N=94)$ were derived from the FRESH cohort, an observational, prospective cohort of high-risk HIV-negative women launched in Durban, South Africa [32]. Specimen collection and sharing were approved by the representative review boards (IRB): Duke University (CHAVI 008 A), Massachusetts General Hospital (FRESH), and National Cancer Institute (RDP). Written informed consent was obtained from all subjects at all study sites and specimen were anonymized by IRB-approved procedures.

\section{Genotyping}

Genomic DNA was extracted using QIAamp DNA Blood Mini Kit (Qiagen) from frozen PBMC pellets, typically obtained from $10 \mathrm{ml}$ of whole blood by
Ficoll density gradient centrifugation. Primers were designed for genespecific PCR amplification and sequencing of IGHG1, IGHG2, and IGHG3 exon coding fragments (Tables S8-S9). PCR amplification was performed separately for each primer pair using Platinum Taq polymerase (Thermo Fisher Scientific) according to the manufacturer's protocol in $10 \mathrm{ul}$ volume reaction with $10-50 \mathrm{ng}$ of genomic DNA and $0.2 \mathrm{uM}$ primers. The cycling parameters were as follows: initial denaturation, $94^{\circ} \mathrm{C}-2 \mathrm{~min} ; 35$ cycles, $94^{\circ} \mathrm{C}-30 \mathrm{sec}, \mathrm{T}_{\text {ann }}$ (Table S1) - $30 \mathrm{sec}, 72^{\circ} \mathrm{C}-1 \mathrm{~min} / \mathrm{kb}$; final extension, $72^{\circ} \mathrm{C}-7 \mathrm{~min}$. Sanger sequencing was performed using standard BigDye protocols and the ABI 3700 instrument (Thermo Fisher Scientific). PCR and sequencing products were purified using AMPure XP and CleanSEQ magnetic beads (Beckman Coulter) according to the manufacturer's protocol. Sequencing data were analyzed using Sequencher software (Gene Codes). Variability in the number of exons encoding the hinge region was analyzed by PCR fragment size resolution using a LabChip GX instrument (Perkin Elmer). Polymorphic positions within each gene were labeled to denote gene name, exon name and nucleotide position within the exon: for example, G1_CH1_68. The Eu numbering system was used for amino acid positions [33] (www.imgt.org). To avoid variant calling errors due to background noise and sample handling, genotyping was repeated for $>10 \%$ of samples, including all variants that were present in single individuals. Table S10 contains all genotyping data generated in this study.

\section{Haplotype analysis}

Haplotypes were built using Haploview 4.1 software (www.broad.mit.edu/ $\mathrm{mpg} / \mathrm{hap}$ loview/) with the lower threshold of $1 \%$. The software automatically calculates SNP frequency and $p$ value for deviation from HardyWeinberg equilibrium, which was not significant for any of the SNPs identified in any of the individual populations.

\section{The 1 kGP data and Fst analysis}

The IGHG exon variant data for the 1kGP-ph3 dataset [21] and the newly assembled high coverage version, 1kGP-30X [22], were extracted from https://www.internationalgenome.org/data-portal/data-collection/grch38 and https://www.internationalgenome.org/data-portal/data-collection/30xgrch38, respectively. The $1 \mathrm{kGP}$ genotypes were obtained using DNA from lymphoblastoid cell lines, which are oligoclonal regarding Ig class switching. The data were processed using Plink v1.9.0, BCFtools v1.9 and VCFtools v0.1.16 [34]. The 1kGP-ph3 data were used for haplotype comparison and the $\mathrm{F}_{\mathrm{ST}}$ analysis. Weir and Cockerham's $\mathrm{F}_{\mathrm{ST}}$ [23] values were calculated for nonsynonymous IGHG SNPs having a global minor allele frequency $>10 \%$ using VCFtools v0.1.16. Empirical $p$ values were determined on the $F_{S T}$ values of 1000 randomly sampled SNPs across all autosomes from the $1 \mathrm{kGP}$-ph3 data [21] for super-population pairs, including AFR, EUR, and EAS. Bonferroni correction was applied to $p$ values to correct for multiple comparisons.

\section{REFERENCES}

1. Vidarsson G, Dekkers G, Rispens T. IgG subclasses and allotypes: from structure to effector functions. Front Immunol. 2014;5:520.

2. Bournazos S, Ravetch JV. Diversification of IgG effector functions. Int Immunol. 2017;29:303-10.

3. Goldberg BS, Ackerman ME. Antibody-mediated complement activation in pathology and protection. Immunol Cell Biol. 2020;98:305-17.

4. Stapleton NM, Einarsdottir HK, Stemerding AM, Vidarsson G. The multiple facets of FcRn in immunity. Immunol Rev. 2015;268:253-68.

5. Lefranc M-P, Lefranc G. The immunoglobulin factsbook, Academic Press: San Diego, 2001.

6. Dard P, Lefranc MP, Osipova L, Sanchez-Mazas A. DNA sequence variability of IGHG3 alleles associated to the main G3m haplotypes in human populations. Eur J Hum Genet. 2001;9:765-72.

7. Lefranc MP, Lefranc G. Human Gm, Km, and Am allotypes and their molecular characterization: a remarkable demonstration of polymorphism. Methods Mol Biol. 2012;882:635-80.

8. Ternant D, Arnoult C, Pugniere M, Dhommee C, Drocourt D, Perouzel E, et al. IgG1 allotypes influence the pharmacokinetics of therapeutic monoclonal antibodies through FcRn binding. J Immunol. 2016;196:607-13.

9. de Taeye SW, Bentlage AEH, Mebius MM, Meesters Jl, Lissenberg-Thunnissen S, Falck D, et al. FcgammaR binding and ADCC activity of human lgG allotypes. Front Immunol. 2020;11:740.

10. Chu TH, Crowley AR, Backes I, Chang C, Tay M, Broge T, et al. Hinge length contributes to the phagocytic activity of HIV-specific lgG1 and IgG3 antibodies. PLoS Pathog. 2020;16:e1008083. 
11. Pandey JP, French MA. GM phenotypes influence the concentrations of the four subclasses of immunoglobulin $G$ in normal human serum. Hum Immunol. 1996;51:99-102.

12. Seppala IJ, Sarvas H, Makela O. Low concentrations of $\mathrm{Gm}$ allotypic subsets $\mathrm{G} 3 \mathrm{mg}$ and G1 $\mathrm{mf}$ in homozygotes and heterozygotes. J Immunol. 1993;151:2529-37.

13. Hassan MS, Islam KB, Hammarstrom L, Smith Cl. Regulation of C gamma 3 expression. Role of switch in the allotype-associated variation of human serum IgG3 levels. J Immunol. 1992;148:2555-62.

14. Oxelius VA, Pandey JP. Human immunoglobulin constant heavy G chain (IGHG) (Fcgamma) (GM) genes, defining innate variants of IgG molecules and B cells, have impact on disease and therapy. Clin Immunol. 2013;149:475-86.

15. Pandey JP, Olsson J, Weidung B, Kothera RT, Johansson A, Eriksson S, et al. An Ig gamma Marker Genotype Is a Strong Risk Factor for Alzheimer Disease, Independent of Apolipoprotein E epsilon4 Genotype. J Immunol. 2020;20:1318-22.

16. Pandey JP, Namboodiri AM, Nietert PJ, Yoshimura R, Hori H. Immunoglobulin genotypes and cognitive functions in schizophrenia. Immunogenetics. 2018;70:67-72.

17. Dugoujon JM, Hazout S, Loirat F, Mourrieras B, Crouau-Roy B, Sanchez-Mazas A. GM haplotype diversity of 82 populations over the world suggests a centrifugal model of human migrations. Am J Phys Anthropol. 2004;125:175-92.

18. Jenkins T, Zoutendyk A, Steinberg AG. Gammaglobulin groups (Gm and Inv) of various Southern African populations. Am J Phys Anthropol. 1970;32:197-218.

19. Calonga-Solis V, Malheiros D, Beltrame MH, Vargas LB, Dourado RM, Issler HC, et al. Unveiling the Diversity of Immunoglobulin Heavy Constant Gamma (IGHG) Gene Segments in Brazilian Populations Reveals 28 Novel Alleles and Evidence of Gene Conversion and Natural Selection. Front Immunol. 2019;10:1161.

20. Brusco A, Cinque F, Saviozzi S, Boccazzi C, DeMarchi M, Carbonara AO. The G4 gene is duplicated in $44 \%$ of human immunoglobulin heavy chain constant region haplotypes. Hum Genet. 1997;100:84-9.

21. Genomes Project C, Auton A, Brooks LD, Durbin RM, Garrison EP, Kang HM, et al. A global reference for human genetic variation. Nature. 2015;526:68-74.

22. Byrska-Bishop M, Evani US, Zhao X, Basile AO, Abel HJ, Regier AA, et al. High coverage whole genome sequencing of the expanded 1000 Genomes Project cohort including 602 trios. bioRxiv 2021. https://doi.org/10.1101/2021.02.06.430068.

23. Weir BS, Cockerham CC. Estimating F-Statistics for the analysis of population structure. Evolution. 1984;38:1358-70.

24. Belsare S, Levy-Sakin M, Mostovoy Y, Durinck S, Chaudhuri S, Xiao M, et al. Evaluating the quality of the 1000 genomes project data. BMC Genomics. 2019;20:620.

25. Atherton A, Armour KL, Bell S, Minson AC, Clark MR. The herpes simplex virus type 1 Fc receptor discriminates between IgG1 allotypes. Eur J Immunol. 2000;30:2540-7.

26. Pandey JP, Namboodiri AM, Radwan FF, Nietert PJ. The decoy Fcgamma receptor encoded by the cytomegalovirus UL119-UL118 gene has differential affinity to IgG proteins expressing different GM allotypes. Hum Immunol. 2015;76:591-4.

27. Richardson SI, Lambson BE, Crowley AR, Bashirova A, Scheepers C, Garrett N, et al. lgG3 enhances neutralization potency and Fc effector function of an HIV V2specific broadly neutralizing antibody. PLoS Pathog. 2019;15:e1008064.

28. Michaelsen TE, Aase A, Westby C, Sandlie I. Enhancement of complement activation and cytolysis of human IgG3 by deletion of hinge exons. Scand J Immunol. 1990;32:517-28.

29. Sandlie I, Aase A, Westby C, Michaelsen TE. C1q binding to chimeric monoclonal lgG3 antibodies consisting of mouse variable regions and human constant regions with shortened hinge containing 15 to 47 amino acids. Eur J Immunol. 1989;19:1599-603.

30. de Bakker PI, Raychaudhuri S. Interrogating the major histocompatibility complex with high-throughput genomics. Hum Mol Genet. 2012;21:R29-36.

31. Apps R, Qi Y, Carlson JM, Chen H, Gao X, Thomas R, et al. Influence of HLA-C expression level on HIV control. Science. 2013;340:87-91.

32. Ndung'u T, Dong KL, Kwon DS, Walker BD. A FRESH approach: Combining basic science and social good. Sci Immunol 2018; 3. https://www.science.org/doi/epdf/ 10.1126/sciimmunol.aau2798.

33. Edelman GM, Cunningham BA, Gall WE, Gottlieb PD, Rutishauser U, Waxdal MJ. The covalent structure of an entire gammaG immunoglobulin molecule. Proc Natl Acad Sci USA. 1969;63:78-85.
34. Danecek P, Auton A, Abecasis G, Albers CA, Banks E, DePristo MA, et al. The variant call format and VCFtools. Bioinformatics. 2011;27:2156-8.

\section{ACKNOWLEDGEMENTS}

We thank Ms. Anna Danilenko for technical assistance and Dr. Maureen P. Martin for helpful discussions. We also thank the donors, clinical personnel and investigators involved in the FRESH and CHAVI studies as well as the Research Donor Program at the Frederick National Laboratory for Cancer Research.

\section{AUTHOR CONTRIBUTIONS}

$A A B, D G A, N V$, and $C O$ designed the study; KLD collected specimens; $A A B, M A$, and DGA performed genotyping; $A A B, W Z, N V$, and DGA analyzed the data; $A A B$ and $M C$ drafted the manuscript; $M C$ supervised and directed the project.

\section{FUNDING}

This project has been funded in whole or in part with federal funds from the Frederick National Laboratory for Cancer Research, under Contract No. HHSN261200800001E. The content of this publication does not necessarily reflect the views or policies of the Department of Health and Human Services, nor does mention of trade names, commercial products, or organizations imply endorsement by the U.S. Government. This Research was supported in part by the Intramural Research Program of the NIH, Frederick National Lab, Center for Cancer Research.

\section{COMPETING INTERESTS}

The authors declare no competing interests.

\section{ADDITIONAL INFORMATION}

Supplementary information The online version contains supplementary material available at https://doi.org/10.1038/s41435-021-00156-2.

Correspondence and requests for materials should be addressed to Mary Carrington.

Reprints and permission information is available at http://www.nature.com/ reprints

Publisher's note Springer Nature remains neutral with regard to jurisdictional claims in published maps and institutional affiliations.

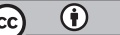

Open Access This article is licensed under a Creative Commons Attribution 4.0 International License, which permits use, sharing, adaptation, distribution and reproduction in any medium or format, as long as you give appropriate credit to the original author(s) and the source, provide a link to the Creative Commons license, and indicate if changes were made. The images or other third party material in this article are included in the article's Creative Commons license, unless indicated otherwise in a credit line to the material. If material is not included in the article's Creative Commons license and your intended use is not permitted by statutory regulation or exceeds the permitted use, you will need to obtain permission directly from the copyright holder. To view a copy of this license, visit http://creativecommons. org/licenses/by/4.0/.

(c) The Author(s) 2021 